\title{
Fluid-Based Biomarkers for Amyotrophic Lateral Sclerosis
}

\author{
Lucas T. Vu ${ }^{1,2} \cdot$ Robert Bowser ${ }^{1,2}$
}

Published online: 8 December 2016

(C) The American Society for Experimental NeuroTherapeutics, Inc. 2016

\begin{abstract}
Amyotrophic lateral sclerosis (ALS) is a highly heterogeneous disease with no effective treatment. Drug development has been hampered by the lack of biomarkers that aid in early diagnosis, demonstrate target engagement, monitor disease progression, and can serve as surrogate endpoints to assess the efficacy of treatments. Fluid-based biomarkers may potentially address these issues. An ideal biomarker should exhibit high specificity and sensitivity for distinguishing ALS from control (appropriate disease mimics and other neurologic diseases) populations and monitor disease progression within individual patients. Significant progress has been made using cerebrospinal fluid, serum, and plasma in the search for ALS biomarkers, with urine and saliva biomarkers still in earlier stages of development. A few of these biomarker candidates have demonstrated use in patient stratification, predicting disease course (fast $v s$ slow progression) and severity, or have been used in preclinical and clinical applications. However, while ALS biomarker discovery has seen tremendous advancements in the last decade, validating biomarkers and moving them towards the clinic remains more elusive. In this review, we highlight biomarkers that are moving towards clinical utility and the challenges that remain in order to implement biomarkers at all stages of the ALS drug development process.
\end{abstract}

Robert Bowser

robert.bowser@dignityhealth.org

1 Department of Neurology, Barrow Neurological Institute, St. Joseph's Hospital and Medical Center, 350 West Thomas Road, Phoenix, AZ 85013, USA

2 Department of Neurobiology, Barrow Neurological Institute, St. Joseph's Hospital and Medical Center, 350 West Thomas Road, Phoenix, AZ 85013, USA
Keywords ALS · Biomarkers · Prognostic · Diagnostic · Clinical $\cdot$ Preclinical

\section{Introduction}

Amyotrophic lateral sclerosis (ALS) is a fatal neurodegenerative disease characterized by the degeneration of both upper and lower motor neurons (MN) in the brain and spinal cord [1, 2]. As the disease progresses, patients exhibit muscle atrophy and consequently lose respiratory function. Only one Food and Drug Administration-approved drug, riluzole, has been shown to extend a patient's lifespan for an average of 2 to 3 months [3-5]. Therefore, there is a critical need to gain further insight into the pathobiology of ALS to further aid in the development of more effective therapeutics.

One of the major challenges associated with ALS pathology stems from its inherent heterogeneity [6]. ALS is a complex disease associated with numerous pathologic mechanisms, including oxidative stress, mitochondrial dysfunction, axonal damage, microglial activation, inflammation, excitotoxicity, and protein aggregation [7-11]. Current diagnostic measures rely upon clinical examination and electrophysiological measurements [6, 12], which, in most cases, have not enabled early diagnosis where potential therapies would likely be most effective. One potential way to improve earlier diagnosis would be to utilize biomarkers specific to ALS that can be identified early in the disease process. In addition, there has been continuous failure of large, latestage clinical trials [13-15]. This highlights the need to enhance preclinical (in vitro and in vivo) studies and ALS clinical trial design, for example through the inclusion of biomarkers throughout the drug development process $[16,17]$.

A biomarker is a characteristic that is objectively measured as an indicator of normal biological processes, pathogenic 
processes, or pharmacological responses to therapeutic intervention [18-21]. To date, biomarker discovery efforts have been performed using various biofluids, including cerebrospinal fluid (CSF), serum, plasma, urine, and saliva [22]. However, the majority of studies have been performed on CSF, serum, and plasma. In this review, we discuss the current literature with regard to discovery and validation of fluidbased ALS biomarkers. While the list of biomarkers presented here is not exhaustive, we intend to discuss biomarkers that have been widely studied and highlight their demonstrated preclinical and clinical applications if available.

\section{Biomarkers in CSF}

The search for biomarkers related to ALS has been performed predominantly using CSF, owing to its intimate interaction with the central nervous system (CNS). In addition, because the CSF is in contact with the $\mathrm{MN}$ in the brain and spine, alterations in the biochemistry of the CSF could be indicative of neuronal injury and/or neurodegeneration, making the CSF an ideal target for biomarker discovery [23, 24].

\section{Neurofilament Proteins}

Neurofilaments are structural proteins of the cytoskeleton that are expressed in neurons and have been investigated as potential biomarkers for ALS and other neurologic diseases. Accumulation of neurofilament proteins has been linked to MN dysfunction [25-27]. Axonal injury releases these proteins into the CSF and subsequently to the blood. Owing to their relative stability in biofluids, detection of neurofilaments is relatively simple and can be performed via immunoassays, making these proteins potential biomarkers of the underlying pathology of ALS. Early studies identified increased phosphorylated neurofilament heavy chain $(\mathrm{pNFH})$ in the CSF of patients with ALS when compared with healthy controls and other neurodegenerative diseases [28, 29]. Additional studies have demonstrated a utility for $\mathrm{pNFH}$ or a ratio of pNFH to complement $\mathrm{C} 3$ in the CSF as a diagnostic marker for ALS [30-33]. pNFH levels in the CSF or blood also have prognostic utility and can be used to assess the rate of disease progression and survival [30, 34], where disease progression is measured via the rate of decline of the ALS Functional Rating Scale-Revised (ALSFRS-R) score. Brettschneider et al. [28] observed decreased survival with high levels of pNFH in CSF; however, no correlation was found with the ALSFRS. Interestingly, higher CSF pNFH was also observed in patients with ALS who exhibited a predominant upper MN involvement, indicating potential use of pNFH in distinguishing upper and lower $\mathrm{MN}$ degeneration. In contrast to these findings, high levels of pNFH and neurofilament light chain (NFL) levels were correlated with central MN involvement [29].
While clinical examination is an effective method to distinguish upper $\mathrm{MN}$ and lower $\mathrm{MN}$ involvement, biomarkers could potentially be used to help monitor progression or to measure the effects of drugs specifically targeting MN within these areas. However, further studies are required to verify neurofilament or any other biomarker to distinguish involvement of upper versus lower MNs.

Many studies have also examined levels of NFL in ALS and control groups [35-38]. Similar to pNFH, increased levels of NFL were correlated with rate of progression, as monitored by the rate of decline in ALSFRS-R scores, as well as upper MN involvement [36, 39]. A rapid time to generation (time of symptom spreading from bulbar or spinal localization to both) has also been noted with increased CSF NFL [37]. Diffusion tensor imaging was recently used to evaluate the correlation between increased CSF NFL levels and white matter damage, where decreased fractional anisotropy and increased radial diffusivity were observed within the corticospinal tracts of patients with ALS but not in healthy controls, reflecting the degeneration of axons and subsequent release of NFL into the CSF [39].

An important question is whether levels of pNFH or NFL change during disease progression within individual patients. Longitudinal blood and CSF studies focusing on levels of NFL were recently evaluated from patients with slow, intermediate, and fast progressing ALS, as defined by the ALSFRS-R rate of decline [38]. In the CSF, NFL levels exhibited a modest $1.6 \%$ increase/month in slow-progressing ALS, whereas those considered as intermediate ALS progressors exhibited little change over a 15 -month period. However, patients with fast-progressing ALS exhibited a $3.3 \%$ increase/month over a 6 -month period. The reason for modest increases in slow progressors but no increase for intermediate progressors is unclear, though may be owing to the small numbers of patients in each group and is worthy of further examination in a much larger patient population. A recent study has also shown increases in CSF NFL correlated with decreases in ALSFRS-R scores [40]. However, no differences between fast-, slow-, and intermediate-progressing ALS was observed with NFL, pNFH, Tau, or pTau [40]. While these results suggest a prognostic biomarker utility of NFL, prior studies have noted inherent instability of NFL in CSF and its susceptibility to protease degradation [41, 42]. Therefore, caution may be exercised with studies involving NFL as a fluid-based biomarker.

Recent longitudinal studies examining pNFH in CSF or blood show relatively stable levels over time, though levels of pNFH in serum appeared to increase at early stages of the disease $[34,43]$. Weydt et al. measured levels of pNFH and NFL in CSF and blood of symptomatic and asymptomatic carriers of disease causing mutations to determine if either biomarker could be detected in symptomatic individuals [44]. While elevated levels were observed in symptomatic 
mutation carriers, neither pNFH nor NFL were detected at elevated levels in asymptomatic ALS mutation carriers. It should be noted that one caveat of this study is that most of the asymptomatic mutation carriers harbored the $C 9$ orf 72 repeat expansion. However, C9orf72 does not have $100 \%$ penetrance and therefore some of these individuals may never develop ALS and thus no changes in pNFH or NFL would be detected, which is consistent with the results from this study. A study in the superoxide dismutase 1 (SOD1) transgenic mouse model of ALS also indicated that pNFH levels increase near or at the time of symptom onset. From current data, we believe that pNFH and NFL levels may significantly increase in patient biofluids only near or upon symptom onset in patients with ALS.

\section{Biomarkers of Inflammation}

A common feature among many neurodegenerative diseases is glial activation accompanied by increased levels of inflammatory mediators, potentiating neuroinflammation and cell death $[45,46]$. Recent proteomic analyses identified 248 proteins in the CSF from both healthy controls and patients with ALS that exhibited enrichment in biologic roles related to the complement cascade and acute inflammation [47]. Additionally, studies in our group using unbiased proteomic analyses of CSF also identified that acute inflammatory responses and complement activation were among the top pathways altered in the CSF of patients with ALS when compared with healthy controls and other neurologic diseases [10]. Therefore, inflammatory mediators present potential biomarkers for ALS. Inflammatory mediators that have been measured in the CSF of ALS and control patients are summarized in Table 1.

Overall, trends from these studies show increases and decreases in the level of proinflammatory and immune response mediators, suggesting that inflammatory pathways in ALS are independently regulated by many factors. While reasons for these observations need clarification, they demonstrate that cytokines and chemokines may play a role in the pathogenesis of ALS. For example, NSC-34 cells treated with CSF from patients with ALS exhibited decreased cell viability and increased lactate dehydrogenase activity [48], suggesting that factors within or released to the CSF contribute to neurodegeneration. Further analyses in the same study also showed that Chit-1 increased expression in microglia upon exposure to CSF from patients with ALS, suggesting its role in microglial activation. A recent study has also shown a positive correlation between CSF and serum interferon (IFN)- $\gamma$ levels and both correlated with disease progression but not with ALSFRS-R scores [57]. Levels of interleukin (IL)-6 also correlated with hypoxic severity [54], suggesting that cytokine levels are dependent on oxygenation, which highlights a link between hypoxia and inflammation in ALS.
Table 1 Inflammatory mediators detected in the cerebrospinal fluid of patients with amyotrophic lateral sclerosis (ALS)

\begin{tabular}{lll}
\hline Biomarker & Key finding & Reference(s) \\
\hline Chit-1 & $\uparrow$ levels and $\uparrow$ activity & {$[48]$} \\
& $\uparrow$ levels & {$[49]$} \\
Prostaglandin E2 & NC & {$[50]$} \\
& $\uparrow$ levels & {$[51,52]$} \\
VEGF & $\uparrow$ levels & {$[53]$} \\
& $\downarrow$ levels & {$[50]$} \\
IL-6 & $\uparrow$ levels & {$[53,54]$} \\
GM-CSF & $\uparrow$ levels & {$[53]$} \\
IL-2 & $\uparrow$ levels & {$[53]$} \\
IL-15 & $\uparrow$ levels & {$[53]$} \\
IL-17 & $\uparrow$ levels & {$[53]$} \\
MIP-1 $\beta$ & $\uparrow$ levels & {$[53]$} \\
FGF & $\uparrow$ levels & {$[53]$} \\
G-CSF & $\uparrow$ levels & {$[53]$} \\
MIP-1 $\alpha$ & $\uparrow$ levels & {$[53]$} \\
MCP-1 & $\uparrow$ levels & {$[53,55]$} \\
IL-10 & $\downarrow$ levels & {$[53]$} \\
IFN- $\gamma$ & $\downarrow$ levels & {$[53]$} \\
IL-8 & $\uparrow$ levels & {$[55]$} \\
Angiogenin & $\downarrow$ levels & {$[50]$} \\
Angiopoietin 2 & NC & {$[50]$} \\
Follistatin & $\downarrow$ levels & {$[56]$} \\
IL-1 $\alpha$ & $\downarrow$ levels & {$[56]$} \\
Kallikrein-5 & $\downarrow$ levels & {$[56]$} \\
\hline & & \\
& &
\end{tabular}

$\downarrow$ and $\uparrow$ represent decreased and increased levels in patients with ALS vs controls respectively

Chit-1 = chitotriosidase $-1 ; \mathrm{NC}=$ no change observed; VEGF = vascular endothelial growth factor; IL = interleukin; GM-CSF = granulocyte macrophage colony-stimulating factor; MIP = macrophage inflammatory protein; FGF = fibroblast growth factor; G-CSF = granulocyte colonystimulating factor; $\mathrm{MCP}=$ monocyte chemoattractant protein 1 ; IFN = interferon

In addition to extracellular cytokines and chemokines as biomarkers for ALS, the presence of activated inflammatory cell types can also serve as a potential biomarkers. Peripheral monocytes and regulatory T cells enter the CNS and can also modulate inflammation during ALS and play key roles in regulating disease progression $[58,59]$. The point of entry of these cells is believed to be either the blood-brain barrier or the choroid plexus [60]. A combination of integrin receptors and inflammatory signaling molecules [IFN- $\gamma, \mathrm{C}-\mathrm{X}-\mathrm{C}$ motif chemokine 10 , tumor necrosis factor (TNF)- $\alpha$ ] regulate trafficking of monocytes across the blood-brain barrier and leukocytes across the choroid plexus to gain access to the CSF for distribution within the parenchyma [60,61]. Further studies of peripheral immune cell trafficking into the CSF and entry into the brain and spinal cord tissue could potentially be explored using flow-assisted cell sorting in order to define the cell types 
involved. These experiments could yield new insights into the pathogenesis and progression of ALS, as well as reveal the intricate intercellular signaling pathways that will allow for the identification of novel therapeutic biomarkers.

\section{C9orf72 Dipeptide Repeat Proteins}

The C9orf72 repeat expansion is the most common genetic cause of familial ALS (fALS) and frontotemporal lobar degeneration (FTLD) [62, 63]. Nuclear export of mRNA containing this repeat expansion can result in non-ATG-mediated translation that has been detected in multiple neurodegenerative diseases [64]. Non-ATG-mediated translation of the C9orf72 repeat expansion yields 5 dipeptide repeat proteins (DPRs) detected in patients with ALS and FTLD [65, 66]. While these DPRs can be exchanged between cells and have been shown to induce neurotoxic effects in model systems [67, 68], the presence of DPR pathology does not correlate with clinicopathologic measures of disease [69]. However, a recent study has also shown that DRPs can induce spontaneous formation and persistence of stress granules [70], which are cytoplasmic bodies that consist of proteins and mRNA that function to limit translation initiation and are a feature of ALS pathobiology [71]. While monitoring DPR pathology within CNS tissue during disease may be difficult, DPRs have been detected in CSF and blood and therefore could be monitored within these biofluids during disease progression and in clinical trials. Therefore, DPRs could be excellent biomarkers for tracking ALS pathology in response to therapeutic intervention. Previous studies have generated immunoassays for DPRs in tissue extracts and more recently in CSF $[72,73]$. Currently, these DPRs have only been detected in patients with ALS or FTLD with the C9orf72 repeat expansion. Further clinical studies will be required and are currently under development to validate this biomarker for future use in C9-based therapies.

\section{Metabolic Biomarkers}

While there is evidence of numerous pathologic mechanisms associated with ALS, excitoxicity continues to be one of the accepted mediators of disease progression and MN death and has been studied extensively [74-76]. Excitoxicity is a result of overstimulation of glutamate receptors, thereby increasing intracellular calcium levels leading to increased cell death. Therefore, it is reasonable to hypothesize that increased levels of extracellular glutamate could contribute to the progression of ALS. This has been addressed in previous studies where increased levels of glutamate were observed in the CSF of patients with ALS compared with controls [77-82]. In contrast to these findings, others have also demonstrated similar glutamate levels in CSF from patients with ALS and controls [83, 84]. Interestingly, another study shows lower levels of glutamate in the CSF that fails to correlate to disease progression or decreased MN cell death [85]. However, measurements of CSF concentrations of glutamate in response to therapeutics remains to be explored and therefore further investigation is warranted.

Mass spectrometry-based metabolomics has been used to discover metabolic signatures in patients with ALS. Wuolikainen et al. examined the CSF of patients with ALS with mutations in the SOD1 gene and identified distinct metabolic profiles for particular SOD1 mutations, with the main differentiating metabolites being arginine, lysine, ornithine, serine, threonine, and pyroglutamic acid [86]. A recent study from the same group combining multiple mass spectrometric methodologies found increased creatine and decreased creatinine levels in the CSF of patients with ALS when compared with controls [87]. While the large phase III clinical trial of dexpramipexole failed to exhibit efficacy, a post hoc analysis found that creatinine loss correlated with disease progression and this loss was reduced in patients treated with dexpramipexole [88]. This result supports continued investigation of patient creatine and creatinine in the blood and CSF as potential prognostic biomarkers for ALS.

\section{TAR DNA-Binding Protein of 43 kDa}

TAR DNA-binding protein of $43 \mathrm{kDa}$ (TDP-43) is a core component of ubiquitinated cytoplasmic inclusions in sporadic ALS (sALS), many forms of fALS, and FTLD [89-91]. In postmortem studies, TDP-43 inclusions are detected in approximately $97 \%$ of patients with ALS. Based on these findings, investigators have pursued TDP-43 as a biofluid biomarker for ALS [92-95]. If changes can be reliably detected in blood or CSF, TDP-43 could be a valuable biomarker for the majority of patients with ALS. Overall, these studies identified increased levels of TDP-43 in CSF from patients with ALS compared with a variety of neurologically diseased and nondiseased controls. Additionally, increased TDP-43 levels were also observed among patients who were examined within 10 months of disease onset, suggesting that TDP-43 might be useful as a prognostic indicator for early stages of ALS [93]. However, the absolute levels of TDP-43 measured in CSF varies across the studies, suggesting that the TDP-43 immunoassays are inconsistent for measuring the protein within CSF or blood samples. One challenge may be that as TDP-43 is a self-aggregating protein, it may self-assemble and generate structures that limit access of the detection antibody when present in biofluids. In addition, TDP-43 has been shown to be subject to alternative splicing causing truncations of the $\mathrm{N}$ - or $\mathrm{C}$-terminus, leading to splice variants linked to ALS [96]. TDP-43 has been detected as 2 distinct species via Western blot: a $45-\mathrm{kDa}$ (phosphorylated form) and 28-kDa isoform [95]. However, the $28-\mathrm{kDa}$ isoform was determined to be nonspecific binding to IgG light chain, indicating antibody cross-reactivity. While interest remains high to monitor 
levels of TDP-43 in CSF or blood as a biomarker, significant improvements in TDP-43 assay methodologies are necessary in order to attain this goal.

\section{Cystatin C}

Previous efforts in our group, as well as others, identified cystatin $\mathrm{C}$ as a potential biomarker in the CSF of patients with ALS [49, 97-100]. Cystatin C is a cysteine protease inhibitor that is involved in extracellular matrix (ECM) regulation, as well as a variety of CNS diseases [101, 102]. Within the context of ALS, cystatin $\mathrm{C}$ has been identified as a component of bunina bodies, which are small eosinophilic inclusions in the lower MN [103]. Additionally, a reduction in cystatin C levels in spinal $\mathrm{MN}$ and astrocytes has also been correlated with the formation of TDP-43 inclusions [104]. Using surfaceenhanced laser desorption/ionization, reduced levels of cystatin $\mathrm{C}$ were observed in CSF from patients with ALS as compared with control subjects (healthy and diseased) [97]. Levels of cystatin $\mathrm{C}$ positively correlated with survival time for patients with limb onset but did not correlate with the disease duration [98]. A longitudinal study was also performed on CSF and plasma over a 1 to 2-year time period [105]. In this study, analyses of cystatin C levels in the CSF showed that fast and slow progressors exhibited trends towards decreased and increased levels over time, respectively. However, only the trend in the slow progressors was significant. In contrast to these findings, plasma levels of cystatin $\mathrm{C}$ were significantly higher in patients with ALS as compared with healthy controls [105]. While protein levels can provide insight into the physiology and pathology, protein levels do not necessarily correlate with enzymatic activity, highlighting a significant limitation to the studies performed to date. To address this, one study measured activity of cystatin C; however, no difference in activity was determined between ALS and control groups [106]. Therefore, further studies are needed in order to determine how decreased cystatin $\mathrm{C}$ levels relate to ALS pathogenesis and disease progression and to determine the use of cystatin $\mathrm{C}$ as a biomarker for ALS.

\section{MicroRNA}

MicroRNAs (MiRNAs) are 20 to 25 nucleotide-long noncoding transcripts that regulate biological processes via mRNA cleavage or translational repression of mRNA [107, 108]. During canonical miRNA biogenesis [109], miRNAs are synthesized from primary miRNAs, which are transcribed in the nucleus. Primary mRNAs are processed into pre-miRNAs by Drosha and subsequently exported to the cytoplasm. PremiRNAs are finally processed by the Dicer complex, resulting in the formation of mature miRNA, which form RNA-induced silencing complexes. There are over 1000 miRNAs in humans and they are typically measured using either RNA sequencing, microarray, Nanostring, or real-time quantitative polymerase chain reaction methodologies. Alterations in miRNA regulation has been implicated in a wide variety of CNS disorders [110-112]. Defects in miRNA biogenesis, for example via knockout of Dicer, has been correlated with decreased motor activity and survival, muscle atrophy, denervation, spinal cord sclerosis, and axonopathy in mice [113]. Additionally, previous studies have demonstrated decreased miRNA expression in the ventral lumbar spinal cord from patients with ALS [114], and also in spinal MN with ALS-causing mutations [115]. These results highlight the potential utility of miRNAs as biomarkers for ALS. Profiling of miRNAs in CSF, serum, and plasma of patients with ALS has been performed in few studies (Table 2), indicating the need for continued investigations and large validation studies for potential miRNA biomarkers.

Overall, similar to the results in tissues from patients with ALS, most miRNAs levels are decreased in the CSF and serum of patients with ALS when compared with healthy and diseased controls. miRNA181a-5p [116], miRNA-143-5p [117], miRNA-574-5p [117], and miRNA-338-3p [118] are the only 4 that have been found to be increased in CSF from patients with ALS. While the physiological roles of most of these miRNAs still remains to be defined within the context of ALS, expression of miRNA-143-5p and miRNA-574-5p was shown to be decreased in lymphoblastoid cell lines (LCL)

Table 2 MicroRNAs (miRNAs) differentially expressed in the cerebrospinal fluid (CSF), serum, and plasma

\begin{tabular}{lllll}
\hline miRNA & CSF & Serum & Plasma & Reference \\
\hline miRNA-15b-5p & $\downarrow$ & NA & NA & {$[116]$} \\
miRNA21-5p & $\downarrow$ & NA & NA & \\
miRNA195-5p & $\downarrow$ & NA & NA & \\
miRNA148a-3p & $\downarrow$ & NA & NA & \\
Let7b-5p & $\downarrow$ & NA & NA & \\
miRNA181a-5p & $\uparrow$ & NA & NA & \\
Let7a-5p & $\downarrow$ & NA & NA & \\
Let7f-5p & $\downarrow$ & NA & NA & \\
miRNA-132-5p & $\downarrow$ & $\downarrow$ & NA & {$[117]$} \\
miRNA-132-3p & $\downarrow$ & $\downarrow$ & NA & \\
miRNA-143-5p & $\uparrow$ & $\downarrow$ & NA & \\
miRNA-143-3p & $\downarrow$ & $\downarrow$ & NA & \\
miRNA-574-5p & $\uparrow$ & NC & NA & \\
miRNA-338-3p & $\uparrow$ & $\uparrow$ & NA & {$[118]$} \\
miRNA-1234-3p & NA & $\downarrow$ & NA & {$[119]$} \\
miRNA-1825 & NA & $\downarrow$ & NA & \\
miRNA-4649-5p & NA & NA & $\uparrow$ & {$[120]$} \\
miRNA-4299 & NA & NA & $\downarrow$ & \\
\hline
\end{tabular}

$\downarrow$ and $\uparrow$ represent decreased and increased levels in patients with ALS $v s$ controls, respectively

$\mathrm{NA}=$ miRNA was not measured in the biofluid; $\mathrm{NC}=$ no change observed 
expressing ALS mutant forms of TDP-43, C9 orf 72, and FUS, as well as in sALS-derived LCLs. miRNA-143-5p expression was also decreased in SOD1 mutant LCLs. These results suggest a link between levels of miRNAs and the different forms of ALS (familial and sporadic), making these viable biomarkers. One challenge with the use of miRNAs as biomarkers stems from the fact that 1 miRNA acts on multiple downstream targets. Therefore, the effects of most miRNAs require in-depth studies to elucidate which downstream targets or pathways are activated or inhibited. Clarifying these targets would provide a means for validation for specific miRNAs as potential ALS prognostic or diagnostic biomarkers. In addition, multiple studies often identify different or overlapping miRNAs as ALS biomarkers, highlighting the need for large validation studies to determine which miRNAs represent optimal biomarkers for ALS. It may also be necessary to combine miRNA signatures with other genetic risk factors to optimize biomarkers for subsets of patients with ALS.

\section{Cu/Zn SOD1}

SOD1 is an ubiquitously expressed antioxidant enzyme that is involved in the conversion of superoxide to oxygen and hydrogen peroxide, protecting cells from oxidative stress. Nearly $20 \%$ of fALS cases are caused by SOD1 mutations and has been widely studied [121-123], though this represents a minor fraction of all patients with ALS. One study measured levels of CSF SOD1 levels between patients with ALS and neurologic disease controls and failed to find significant differences between the groups, though significantly higher levels of SOD1 were detected in the CSF of male patients with ALS, suggesting a potential gender stratification biomarker [124]. This study indicated that SOD1 CSF level is not a diagnostic biomarker for ALS. Prior animal studies have shown that silencing $S O D 1$ led to preservation of grip strength and a delay in the onset of motor function deficits $[125,126]$. In a clinical trial for pyrimethamine, an antimalarial drug, SOD1 levels in the CSF decreased along with a trend towards declining Appel ALS scores, a measure of disease progression [127]. In addition, it was shown that while the baseline levels of SOD1 in the CSF varied between patients with ALS, the level remains relatively constant over time in individual patients [128]. These studies suggest that SOD1, while not a diagnostic biomarker, could function as a pharmacodynamic (PD) biomarker for SOD1 therapies that reduce SOD1 levels in the CNS. Recent studies have confirmed this hypothesis, as treatment with SOD1 antisense oligonucleotides greatly reduces SOD1 levels in the CSF in mice [128], thus providing strong support that measurements of SOD1 in the CSF will function as a pharmacodynamic biomarker for antisense treatments that are currently in clinical trials. Results from a phase I clinical trial of intrathecal delivery of SOD1 antisense oligonucleotides in patients with ALS with SOD1 mutations demonstrated safety for this treatment, though no substantial longitudinal changes in SOD1 CSF levels were detected [129]. Additional ALS clinical trials using SOD1 antisense oligonucleotides are currently in development and will likely include SOD1 measures as a PD biomarker of drug treatment and activity.

\section{Biomarkers in Blood (Serum and Plasma)}

While studies involving the CSF have identified many potential ALS biomarkers, mass transfer occurs between the blood and CSF at the blood-CSF barrier, predominantly in the subarachnoid space and the choroid plexus [130,131], suggesting that the same biomarkers could be present in both fluid types. This was previously demonstrated where several studies have indicated significantly high correlations between blood (serum and plasma) and CSF levels of the same biomarker [34, $38,44,118]$. However, other studies have also demonstrated that levels of biomarkers, that were discovered in blood, did not correlate with the levels of that same biomarker found in the CSF, suggesting that these 2 fluids are independently regulated $[105,117]$. Blood-based biomarkers may also be generated by other organ systems affected during ALS, such as peripheral blood cells or degenerating muscle. Therefore, blood is an excellent biofluid for discovery and validation of biomarkers for ALS. Additionally, the lack of ethical implications and decreased difficulty of obtaining blood as compared with CSF makes blood a more viable option.

\section{pNFH and NFL}

With the success in validating neurofilament proteins as ALS biomarkers in the CSF, pNFH and NFL have also been examined as blood-based biomarkers in the serum and plasma. Higher levels of plasma pNFH were observed in patients with ALS as compared with healthy controls, but no difference was observed when compared to diseased controls [31]. Longitudinal studies have confirmed this finding and have also demonstrated stable levels over a 4-month study [132]. In a study consisting only of patients with ALS, high serum levels of pNFH was associated with rapid decline of ALSFRS-R scores at 4 months from baseline measurements but not 12 months [34]. However, higher plasma levels of pNFH were not correlated with decline in ALSFRS-R scores. In contrast to these findings, higher baseline levels of pNFH have been weakly correlated with the decline in ALSFRS-R scores [132]. Interestingly, increased levels of pNFH in plasma and serum was correlated with shorter survival time and bulbar onset [34]. Overall, these results highlight the utility of pNFH for prognosis and potential use in patient stratification.

In whole blood, levels of NFL were significantly higher in patients with ALS as compared with both healthy controls and patients who were asymptomatic but carried mutations in 
C9orf72, SOD1, TARDP, and FUS [44]. Levels of NFL have also been shown to increase in serum and plasma of patients with ALS as compared with controls [38]. In this same study, no increases in plasma levels were observed over 15 months in fast, slow, and intermediately progressing ALS; however, a $4.6 \%$ increase per month was observed in the serum of patients with fast-progressing ALS. In contrast to these studies, measurements of NFL in the sera from patients with diseased $\mathrm{MN}$ could not be accurately quantified owing to significant variation between samples [40].

Additional studies using increased number of blood samples from patients with ALS and disease controls are needed to validate the above findings and to compare directly pNFH to NFL in the same samples. Ideally, these studies should also include CSF to determine the correlation between CSF and blood levels of pNFH and NFL. This type of head-to-head comparison will determine if one neurofilament protein is superior to the other, or if a particular neurofilament protein is best for identifying or monitoring a specific subset of the ALS population.

\section{miRNAs}

In addition to miRNAs in the CSF, investigations in the serum and plasma have also been performed (Table 2) [119, 120, 133]. Significant decreases in miRNA-1234-3p were only observed in sera from patients with sALS compared with healthy controls, but no significant difference was observed in sera from patients with fALS as compared with healthy controls [119]. This suggests that miRNA-1234-3p may allow for specific diagnosis of sALS. Plasma miRNA-4649-5p has been shown to increase in patients with sALS and was shown to be negatively correlated with disease duration [120]. miRNA424 and miRNA-206 had significantly higher expression in ALS plasma compared with healthy controls [133]. Interestingly, levels of miRNA-424 correlated with the medical research council sum score, which is a measure of lower $\mathrm{MN}$ integrity, indicating its potential use in patient stratification. To the best of our knowledge, there have not been any studies that have used miRNA as a secondary marker for therapeutic development, and thus further studies are warranted.

\section{Biomarkers of Inflammation}

Similar to the results in CSF, increases in levels of inflammatory factors (IL-6 and IL-8) were also observed [134]. However, in contrast to levels in the CSF, decreases in immune factors (IL-5 and IL-2) and glutathione levels were also shown indicating decreased immune response with increased oxidative stress. In addition to inflammatory factors, levels of the anti-inflammatory factor transforming growth factor (TGF)- $\beta 1$ were shown to increase in plasma from patients with ALS as compared with controls and was positively correlated with disease duration [135]. Similar observations were also observed in serum where TGF- $\beta 1$ concentrations were higher in patients with ALS than in controls [136]. Given that one of the pathological mechanisms of ALS is inflammation, these findings seem counterintuitive to what would be expected as TGF- $\beta 1$ is an anti-inflammatory mediator and thus should attenuate neuroinflammation. Nevertheless, these findings suggest that TGF- $\beta$ could potentially modulate ALS progression and therefore further investigations will be required in order to determine the downstream effects and participation of this cytokine in the pathogenic process. Identification of prognostic inflammatory biomarkers has also been performed using plasma. Increases in creatine kinase, ferritin, TNF- $\alpha$, IL$1 \beta$, IL-2, IL-8, IL-12p70, IL-4, IL-5, IL-10, and IL-13, and decreases in IFN- $\gamma$, have been observed [137]. Longitudinal analyses revealed that plasma IL-6 increased over 6 visits in those that had slow progressing ALS, those that are male, exhibited limb onset, less functional impairment, and also in patients with ALS treated with riluzole, further suggesting the utility of IL- 6 in patient stratification. Median levels of TNF- $\alpha$ have also been shown to increase in the plasma of patients with ALS compared with healthy controls, and these elevated levels were observed over an 80-month period [138], implicating prognostic use of this inflammatory marker.

In addition to extracellular cytokines and chemokines, peripheral monocytes can enter the CNS and be detected in postmortem tissues from patients with ALS [139]. Regulatory $\mathrm{T}$ cells within the blood also enter and modulate inflammation within the CNS where reduced expression of FoxP3, a transcription factor required for regulatory T-cell function, correlated with the rate of disease progression and survival of patients [140]. Therefore, flow cytometric analysis of blood-borne T cells and subsequent measurement of FoxP3 levels may provide another prognostic indicator of disease progression.

\section{TDP-43}

Levels of TDP-43 have been assessed in plasma where increased levels were observed in patients with ALS compared with controls [141]. Longitudinal analyses showed consistent levels over time in most patients; however, 1 patient exhibited increased levels over time, potentially highlighting the heterogeneity of the disease. Another study has shown increased levels of phosphorylated TDP-43 in the plasma and CSF in patients carrying the $C 9$ orf 72 expansion [142]. In contrast to these findings, levels of TDP-43 in circulating lymphomonocytes were approximately the same in TDP-43 mutant-carrying cells, nonmutant cells, and controls [143]. However, given the limitations of the current TDP-43 immunoassays as described above, the potential biomarker utility of TDP-43 in blood remains uncertain. 


\section{Metabolic Biomarkers}

Previous screening of amino-acid concentrations in plasma have shown increased levels of glutamate and lysine in patients with early-onset ALS, while leucine levels decreased [144]. Increased levels of plasma glutamate were positively correlated with disease duration [145], and was observed in patients who exhibited spinal onset [146]. Interestingly, while riluzole decreased disease progression, no effect on the levels of plasma glutamate or glycine was observed after 6 months of treatment [146]. This is in contrast to what has been shown in serum, where glutamate and total amino-acid concentrations were decreased after a 6-month treatment with riluzole [147]. The reasons for these discrepancies are not known. Nevertheless, these results demonstrate the utility of glutamate as a biomarker for ALS in serum in response to drug intervention.

Paganoni et al. also demonstrated that serum uric acid levels correlate with survival in men with ALS [148]. This study measured serum uric acid levels in a large number of ALS clinical trial participants, and by controlling patient body mass index, showed that higher baseline levels were associated with increased survival. The threshold cut-off value for improved survival was $4.8 \mathrm{mg} / \mathrm{dl}$, which, interestingly, was a level detected in only a small fraction of women. Continued validation studies are required for this serum biomarker to determine if a separate, gender-specific cut-off level can be identified for women.

A global metabolomics study of plasma from over 250 subjects identified a 32-member panel of metabolites that differentiated ALS from healthy and disease controls [149]. Included in this biomarker panel were creatine, creatinine, urate, glutamine, and pyroglutamine, all identified in prior or subsequent metabolomics studies. While additional validation studies are needed, these studies suggest that specific metabolic alterations can be identified in patients with ALS that contribute to disease progression and survival.

\section{Biomarkers in Urine}

While CSF and blood-based screening has shown promise in identifying fluid-based biomarkers, the inherently invasive nature of obtaining these fluids, especially with CSF, presents difficulty. Additionally, ethical considerations limit the collection of CSF from healthy patients and, as a result, studies focusing on characterization of CSF from healthy individuals are limited $[150,151]$. One way to circumvent this issue is through investigation of urine-based biomarkers. However, there are a limited amount of studies using urine from patients with ALS.

To the best of our knowledge, only neurotrophin receptor p75 (p75NTR) [152], glucosylgalactosyl hydroxylysine (Glu-
Gal Hyl) [153], type IV collagen [154], and 8-hydroxyl-2'deoxyguanosin $(8 \mathrm{OH} 2$ 'dG) $[155,156]$ have been explored as potential biomarkers warranting further studies of urine for ALS biomarkers. Recently, the extracellular domain of p75NTR, which is a regulator of cell survival and death, was shown to exhibit increased levels in urine from ALS patients with limb or bulbar onset as compared with healthy controls [152]. Similar trends were also observed in $\mathrm{SOD} 1{ }^{\mathrm{G} 93 \mathrm{~A}}$ mice [152]. The p75NTR results using human urine also correlated with a decline in ALSFRS-R scores, such that patients with fast-progressing ALS exhibited higher levels of p75NTR. Degradation of collagen results in the excretion of the metabolites Glu-Gal Hyl and galactosyl hydroxylysine into urine, making these feasible biomarkers for ALS [153]. Glu-Gal Hyl exhibited decreased levels in ALS which continually decreased throughout the duration of the disease, while no change in galactosyl hydroxylysine levels were observed. These results correlate with earlier studies where total collagen levels also decreased in lateral corticospinal tract and anterior horn in the spinal cord of patients with ALS [157]. Decreases in type IV collagen levels in urine were also observed in patients with ALS as compared with diseased and healthy controls [154]. These results align with the established paradigm of ECM turnover during the progression of ALS. In addition to structural proteins, levels of $8 \mathrm{OH} 2$ 'dG, a marker of DNA damage due to oxidative stress, were elevated in patients with ALS as compared with healthy and diseased controls [155]. The change in urine levels of $8 \mathrm{OH} 2$ ' $\mathrm{dG}$ were negatively correlated with the rate of change of ALSFRS-R scores, suggesting that $8 \mathrm{OH} 2$ 'dG could serve as a potential prognostic marker, and also confirms that oxidative stress contributes to the ALS pathology. Similar observations were shown in another study using patients with sALS; however, no trend was observed between $8 \mathrm{OH} 2$ 'dG levels and ALSFRS-R scores [156]. Taken together, these results demonstrate the potential utility of urine for ALS biomarker discovery. Urine-based biomarkers may also provide further insight into the roles of ECM turnover and DNA damage related to ALS pathology.

\section{Biomarkers in Saliva}

Similar to studies involving urine-based biomarkers, there are relatively few studies that have investigated potential biomarkers for ALS in saliva, as shown in Table 3. These studies have focused on markers that are synthesized by the endocrine system, suggesting its potential involvement in the pathogenesis of ALS. However, in one study chromogranin A levels did not correlate with many measures from the ALS assessment questionnaire, such as physical mobility, activities of daily living, eating and drinking, and communication. Conversely, cortisol levels did correlate with accepted 
Table 3 Endocrine amyotrophic lateral sclerosis (ALS) biomarkers in saliva

\begin{tabular}{|c|c|c|c|c|}
\hline Protein & Function & Levels in patients with ALS $v s$ controls & Correlations $( \pm)$ & Reference \\
\hline Chromogranin A & Endocrine stress marker & $\begin{array}{l}\text { Increased (controls = moderate ALS, } \\
\text { vascular dementia, and healthy) }\end{array}$ & + with emotional functioning & {$[158]$} \\
\hline Cortisol & Steroid hormone & Decreased at $30 \mathrm{~min}$ postawakening & $\begin{array}{l}\text { + with ALSFRS and MMT measures- } \\
\text { with depressive status measures }\end{array}$ & {$[159]$} \\
\hline
\end{tabular}

ALSFRS $=$ ALS Functional Rating Scale; MMT = manual muscle testing

measures of ALS progression. Therefore, much further indepth studies using accepted measures of ALS progression such as the ALSFRS-R scores, manual muscle testing, and forced vital capacity are required in order to validate this biomarker, as well as others derived from saliva and their relation to ALS pathogenesis. Nevertheless, given the limited amount of information using this easily obtained biofluid, future studies could focus upon identifying additional biomarkers and increased sample sizes, as well as verification in clinical trials.

\section{Clinical Applications}

Many of the studies discussed previously have demonstrated the diagnostic and prognostic potential for these biomarkers for ALS. In follow-up studies, applying these biomarkers in clinically relevant situations is used for further validation, as summarized in Table 4.

A few studies have already utilized inflammatory mediators as biomarkers during clinical trials [160-162, 164]. Given that inflammation is an enriched process in patients with ALS as compared with controls [47], treatments have been studied that are aimed at reducing inflammatory marker mRNA and protein abundances. Nineteen patients with moderately progressing ALS and lower MN disease, treated with a combination of anakinra and riluzole, were tolerant to the administered doses. However, no effect on disease progression was observed [160]. These patients also demonstrated a slight, yet insignificant, decrease in serum IL- 6 and TNF- $\alpha$ over the trial period. However, fibrinogen levels were significantly decreased in both fluids, while levels of C-reactive protein increased in serum, indicating that this drug combination may target specific inflammatory pathways. Patients with ALS have also been treated with thalidomide but did not show any improvement in ALSFRS-R rate of decline nor did they exhibit any changes in serum TNF- $\alpha$ level over 9 months of treatment [164]. Tocilizumab (Actemra ${ }^{\circledR}$ ) is an antibodybased therapeutic that inhibits IL-6 and soluble IL-6 signaling, and has been shown to decrease mRNA expression and protein secretion of inflammatory mediators in peripheral blood mononuclear cells from patients with sALS alone and in coculture with activated macrophages after $24 \mathrm{~h}$ of treatment [162]. Direct infusion of Actemra into patients has been shown to decrease mRNA expression of many inflammatory cytokines in the serum of patients with sALS with high basal inflammation both acutely and longitudinally, and this correlated with attenuated decrease in ALSFRS-R scores [161]. Further studies utilizing Actemra in phase II clinical trials is

Table 4 List of biomarkers used in preclinical and clinical applications

\begin{tabular}{|c|c|c|c|c|}
\hline Drug or therapy & Mechanism of action & Biomarker(s) used for evaluation & Biofluid & Reference(s) \\
\hline Anakinra + riluzole & IL-1 receptor antagonist & $\begin{array}{l}\text { IL-6, TNF- } \alpha \text {, of fibrinogen levels, } \\
\text { C-reactive protein }\end{array}$ & Serum & {$[160]$} \\
\hline Tocilizumab (Actemra®) & Inhibits IL-6 and soluble IL-6 & $\begin{array}{l}\text { IL-1 } 1 \beta, \text { IL- } 6 \text {, IL-10, GM-CSF, and } \\
\text { TNF- } \alpha \text {, and evaluation of mRNA } \\
\text { levels of many inflammatory } \\
\text { cytokines }\end{array}$ & PBMC & {$[161,162]$} \\
\hline Arimoclomol & Inducer of heat shock response & pNFH & Plasma & [163] \\
\hline Thalidomide & Immune suppression & TNF- $\alpha$ & Serum & {$[164]$} \\
\hline Memantine + Riluzole & NMDA receptor antagonist & Tau and $\mathrm{pNFH}$ & CSF & {$[165]$} \\
\hline $\begin{array}{l}\text { Antisense oligonucleotide } \\
\text { against SOD1 }\end{array}$ & $\begin{array}{l}\text { Short DNA sequence that binds } \\
\text { mRNA and triggers degradation }\end{array}$ & SOD1 & $\mathrm{CSF}$ & {$[128]$} \\
\hline Pyrimethamine & Antimalarial drug & SOD1 & CSF and leukocytes & {$[127]$} \\
\hline Celecoxib & NSAID that inhibits COX-2 & Prostaglandin E2 & $\mathrm{CSF}$ & {$[166]$} \\
\hline Dexpramipexole & Improves mitochondrial efficiency & Creatinine & Plasma & {$[88]$} \\
\hline
\end{tabular}

$\mathrm{IL}=$ interleukin; $\mathrm{TNF}=$ tumor necrosis factor; $\mathrm{GM}-\mathrm{CSF}$ = granulocyte macrophage colony-stimulating factor; PBMC = peripheral blood mononuclear cells; pNFH = phosphorylated neurofilament heavy chain; NMDA = N-methyl-D-asparate; $C S F=$ cerebrospinal fluid; $S O D=$ superoxide dismutase; NSAID $=$ nonsteroidal anti-inflammatory drug; COX = cyclooxygenase 
already planned and will need to be performed in order to validate fully its effects [167].

Prior studies identified up to a 10 -fold increase in the prostaglandin E2 levels in the CSF of patients with ALS as compared with healthy control patients [51, 52]. Prostaglandins, especially prostaglandin E2, are generated by cycolooxygenase (COX) activation and stimulate the release of glutamate by astrocytes. Given the large difference in the levels of this inflammatory mediator in the CSF of patients with ALS, it was evaluated as a secondary endpoint in a clinical trial for celecoxib, a nonsteroidal anti-inflammatory drug that inhibits COX-2 [166]. However, similar prostaglandin E2 levels were observed in both the celecoxib-treated and nontreated groups, though no changes in clinical parameters of disease or survival were observed by drug treatment, and levels were not elevated in the ALS patients. These results suggest that prostaglandin E2 is not a biomarker for ALS.

Early-phase clinical trials have also been performed using pNFH as biomarker of axonal injury and neuronal degeneration. Neurodegeneration and axonal injury releases neurofilament proteins into the CSF and, ultimately, the blood. Therefore, this biomarker has been considered for neuroprotective-based therapies. Preclinical studies utilizing SOD $1^{\mathrm{G} 93 \mathrm{~A}}$ mice demonstrated the use of $\mathrm{pNFH}$ as a biomarker to test the effects of arimoclomol [163]. Plasma levels of pNFH were observed to decrease in these mice over approximately 60 days of treatment. Prognostic utility of pNFH and Tau has been used in a pilot study aimed at assessing the safety and tolerability of a combination of memantine and riluzole [165]. Patients who completed the treatment showed a decrease in the rate of disease progression along with a trend toward decreases in CSF levels of both Tau and pNFH. Further studies utilizing increased samples sizes are needed to validate these results. Nevertheless, this demonstrates the application of pNFH as a biomarker for therapeutic interventions.

Clinical trials have also demonstrated the use of SOD1 as a biomarker for ALS $[127,128]$. SOD1 mutations are common among many patients with fALS and are a contributing factor to increased oxidative stress. As a result, therapies have been developed and tested that are aimed at decreasing levels of SOD1. Winer et al. measured SOD1 levels in the frontotemporal cortex and in the CSF as a biomarker for SOD1 antisense oligonucleotide therapy in rats and demonstrated a decrease in SOD1 levels, in both cases, as compared with untreated controls [128]. Interestingly, in humans, SOD1 levels in the CSF did not correlate with disease severity or progression, but these levels did remain relatively stable with repeat measurements, which suggests its potential utility as a PD biomarker for antisense-based therapies. In contrast, the antimalarial drug, pyrimethamine, has also been shown to decrease levels of SOD1 in leukocytes and the CSF; however, this was only shown in 2 patients [127]. While these trials demonstrate SOD1 as a potential prognostic biomarker, further studies with increased samples sizes are still needed.

\section{Current Status and Future Directions}

With respect to the studies reviewed here, many promising biomarkers have demonstrated diagnostic and prognostic utility for ALS. However, some of these biomarkers may not be sensitive and/or specific enough to segregate patients with ALS from neurologic disease or healthy controls. Table 5 lists results from the top 5 specific biomarkers with published data on sensitivity and specificity for ALS.

The vast majority of the biomarkers listed have been studied in the CSF, highlighting this biofluid in the discovery of biomarkers related to ALS. All of the biomarkers listed in Table 5 have been studied with adequate sample sizes and have demonstrated both high sensitivities and specificities for ALS. To date, the most advanced biofluid-based biomarkers for ALS are pNFH and NFL. These proteins appear as soon as symptoms are present, are inherently stable proteins in the blood and CSF, and correlate with the rates of disease progression and survival. Additionally, these proteins are highly abundant and changes in levels over time due to neurodegeneration are easily detected by immunoassays. Multicenter studies have also validated pNFH and NFL, something not yet accomplished for any other biofluid-based ALS biomarker. Additionally, neurofilament-based immunoassays are being moved into the clinic by Iron Horse Diagnostics, Inc. as an aid for earlier diagnosis, for prognostic indicators of ALS, and to monitor drug effects in clinical trials that attempt to reduce neurodegeneration. While studies on p75NTR are somewhat limited, its discovery in urine makes it a promising fluid-based biomarker for ALS for continued investigation. Further studies are needed to confirm its sensitivity and specificity for ALS in larger numbers of patients with ALS and disease controls.

One of the more interesting approaches to biomarker discovery is using combinations or ratios of biomarkers from different pathogenic pathways, which has been shown to increase the sensitivity and/or specificity of potential ALS biomarkers [31, 39, 116, 168]. For example, as mentioned previously, studies from our group have shown that a ratio of $\mathrm{pNFH} / \mathrm{C} 3$, which combines data representing both axonal degeneration and inflammatory processes, maintains the sensitivity and slightly increases the specificity for distinguishing ALS from controls compared with pNFH alone (see Table 5) [31]. Using vector machine algorithms, combinations of IL10, IL-6, granulocyte macrophage colony-stimulating factor, IL-2, and IL-15 not only exhibited adequate sensitivities and specificities, but have also demonstrated $89 \%$ accuracy when using these 5 biomarkers to distinguish patients with ALS 
Table 5 Top 5 fluidbased biomarkers that have been used to distinguish patients with amyotrophic lateral sclerosis (ALS) from diseased and nondiseased controls

\begin{tabular}{llllll}
\hline Biomarker & Biofluid & Patient information & $\begin{array}{l}\text { Sensitivity } \\
(\%)\end{array}$ & $\begin{array}{l}\text { Specificity } \\
(\%)\end{array}$ & Reference \\
\hline pNFH & CSF & 40 ALS, 40 non-ALS DC & 97.3 & 83.8 & {$[49]$} \\
pNFH & CSF & 253 MND, 85 DC & 83.0 & 80.0 & {$[40]$} \\
pNFH & CSF & 71 ALS, 52 DC, 40 HC & 87.7 & 93.7 & {$[31]$} \\
pNFH/C3 & CSF & 71 ALS, 52 DC, 40 HC & 87.7 & 94.6 & {$[31]$} \\
NFL & CSF & 254 MND, 85 DC & 77.0 & 88.0 & {$[40]$} \\
NFL & CSF & 64 ALS, 36 HC & 97.0 & 95.0 & {$[38]$} \\
NFL & Serum & 64 ALS, 36 HC & 89.0 & 75.0 & {$[38]$} \\
NFL & Plasma & 103 ALS, 42 HC & 90.0 & 71.0 & {$[38]$} \\
Combination of IL-10, IL-6, & CSF & 41 ALS, 33 DC & 87.5 & 91.2 & {$[53]$} \\
GM-CSF, IL-2, and IL-15 & Urine & 28 ALS, 12 HC & 93.0 & 100.0 & {$[152]$} \\
p75NTR & Urine & 28 ALS, 19 DC & 93.0 & 79.0 & {$[152]$} \\
p75NTR & & & & & \\
\hline
\end{tabular}

pNFH = phosphorylated neurofilament heavy chain; $\mathrm{CSF}=$ cerebrospinal fluid; $\mathrm{DC}=$ diseased controls; $\mathrm{MND}=$ patients with motor neuron disease; $\mathrm{HC}=$ healthy controls; NFL = neurofilament light chain; IL = interleukin; GM-CSF = granulocyte macrophage colony-stimulating factor; p75NTR $=$ neurotrophin receptor $\mathrm{p} 75$ from neurologically diseased controls [53]. In a subsequent study from the same group, mathematical multivariate modeling was employed [20]. These models included a variety of inflammatory cytokines, growth factors, and proteins involved in iron metabolism, as an accurate measure of predicting disease duration in both CSF and plasma with coefficient of determination $\left(\mathrm{R}^{2}\right)$ values of 0.617 and 0.769 , respectively, indicating that a combination of factors can be used for prognosis. Combining the 2 models into 1 comprehensive model that includes both CSF and plasma factors improved the $\mathrm{R}^{2}$ value to 0.962 . Another study identified a negative correlation between CSF and serum NFL levels with fractional anisotropy measurements [39], demonstrating the potential use of both protein levels with neuroimaging measurements. Ratios of miRNA181a-5p/miRNA15b-5p and miRNA181a-5p/miRNA-21-5p showed a considerable improvement in specificity with a slight decrease in sensitivity compared with each individual miRNA [116]. However, studies with increased sample sizes would be beneficial and are needed to verify these results. Taken together, these studies highlight the need for routinely measuring sensitivity and specificity for potential ALS biomarkers and it would be beneficial if appropriate disease controls are used in the study. To date, few studies have attempted to combine biomarkers across multiple biofluids (blood, CSF, urine), and this represents another area for future investigation. Studies should not only combine biofluid-based biomarkers, but also genetic risk factors and/or imaging-based biomarkers to optimize patient stratification or patient selection for specific diseasemodifying therapies.

While many other biomarkers discussed here have shown initial promise, they must be further validated by multiple independent laboratories or via collaborative multicenter studies. In addition, assays used to measure these biomarkers must be optimized and validated in order to move toward clinical utility. Biomarkers should also be incorporated into all stages of ALS drug development, from preclinical studies to early-stage and, ultimately, late-stage clinical trials. Biomarkers should be identified that permit demonstration of drug-target engagement and downstream pathway effects. In addition, the inclusion of disease progression biomarkers will also help demonstrate therapeutic efficacy. These biomarkers should also augment the current clinical measures used in ALS clinical trials. While a few small ALS clinical trials have incorporated biofluid-based biomarkers to monitor drug effects, it should be noted that these studies have shown limited, and even in some cases no, drug efficacy, which, consequently, does not permit proper evaluation of these biomarkers for monitoring drug action. Use of SOD1 or DPR measurements in the CSF as PD biomarkers for clinical trials of SOD1 antisense or anti-C9orf72-directed therapies may have more immediate impact.

One area receiving greater attention is determination of the change in biomarker levels over time within individual patients (longitudinal studies). Some examples of these approaches, albeit from targeted analyses, have been performed [38, 105, 169], and have been discussed throughout this review. These studies require large sample sizes consisting of patients with ALS, neurologic disease controls, and healthy controls, in order to assess how particular biomarkers change over time. New longitudinal studies aimed at discovering changes in biomarker levels using unbiased methodologies will identify new biomarkers that track specific patient subpopulations during disease progression, as well as new therapeutic targets.

Finally, one other biomarker discovery method is a comprehensive screening of metabolites in each of these biofluids 
[170]. Many untargeted metabolite studies have been previously performed in CSF [171, 172], plasma [149, 173], and serum [174], but metabolite identification is usually confined to searches using in-house databases, which biases the results. Further untargeted studies could yield deeper insights into metabolic dysregulation associated with ALS. One of the challenges of an untargeted metabolomic study is the lack of a comprehensive metabolite database, which limits the use of automated algorithms [175]. The most comprehensive human metabolomics databases to date is the human metabolome database [176]. For those metabolites that are not in the database, researchers rely on de novo sequencing, which may not be feasible for many in-depth untargeted metabolomics analyses. Further development of these databases would provide another avenue for biomarker discovery in biofluids for all diseases.

While ALS biomarker discovery efforts have been quite successful in the last decade, continued efforts are necessary to appropriately validate candidate biomarkers and the assays used to measure them. Incorporation of biomarkers in all stages of ALS drug development will vastly improve our ability to test drugs properly in clinical trials and, ultimately, find therapeutic treatments for ALS. With increased efforts, the hope is that a biomarker will not only aid in making faster Go/No-Go decisions in clinical trials, but also act as surrogate markers for primary endpoints in late-stage clinical trials. For now, the field of ALS biomarkers remains an active area of investigation with much promise as biomarkers are reaching clinical utility.

Acknowledgments This work was supported by National Institutes of Health/National Institute of Neurological Disorders and Stroke grants NS068179 and NS061867, and ALS Association funding to RB.

Required Author Forms Disclosure forms provided by the authors are available with the online version of this article.

\section{References}

1. Kiernan MC, Vucic S, Cheah BC, et al. Amyotrophic lateral sclerosis. Lancet 2011;377(9769):942-955.

2. Cleveland DW, Rothstein J. From Charcot to Lou Gehrig: deciphering selective motor neuron death in ALS. Nat Rev Neurosci 2001;2:806-819.

3. Bensimon G, Lacomblez L, Meininger V, Group ARS. A controlled trial of riluzole in amyotrophic lateral sclerosis. N Engl J Med 1994;330:585-591.

4. Lacomblez L, Bensimon G, Leigh PN, Guillet P, Meininger V, II ARSG. Dose-ranging study of riluzole in amyotrophic lateral sclerosis. Lancet 1996;347:1425-1431.

5. Miller RG, Mitchell JD, Moore DH. Riluzole for amyotrophic lateral sclerosis (ALS)/motor neuron disease (MND). Cochrane Database Syst Rev 2012(3):CD001447.

6. Bowser R, Turner MR, Shefner J. Biomarkers in amyotrophic lateral sclerosis: oppportunities and limitations. Nat Rev Neurol 2011;7:631-638.
7. Bruijn LI, Miller TM, Cleveland DW. Unraveling the mechanisms involved in motor neuron degeneration in ALS. Annu Rev Neurosci 2004:27:723-749.

8. Shaw PJ. Molecular and cellular pathways of neurodegeneration in motor neurone disease. J Neurol Neurosurg Psychiatry 2005;76(8):1046-1057.

9. Sabatelli M, Conte A, Zollino M. Clinical and genetic heterogeneity of amyotrophic lateral sclerosis. Clin Genet 2013;83(5):408416.

10. Collins MA, An J, Hood BL, Conrads TP, Bowser RP. Label-free LC-MS/MS proteomic analysis of cerebrospinal fluid identifies protein/pathway alterations and candidate biomarkers for amyotrophic lateral sclerosis. J Proteome Res 2015;14(11):4486-4501.

11. Wijesekera LC, Leigh PN. Amyotrophic lateral sclerosis. Orphan J Rare Dis 2009;4:3.

12. Rutkove SB. Clinical measures of disease progression in amyotrophic lateral sclerosis. Neurotherapeutics 2015;12(2):384-393.

13. Cudkowicz ME, Titus S, Kearney M, et al. Safety and efficacy of ceftriaxone for amyotrophic lateral sclerosis: a multi-stage, randomised, double-blind, placebo-controlled trial. Lancet Neurol 2014;13(11):1083-1091.

14. Cudkowicz ME, Van den Berg LH, Shefner JM, et al. Dexpramipexole versus placebo for patients with amyotrophic lateral sclerosis (EMPOWER): a randomized, double-blind, phase 3 trial. Lancet Neurol 2013;12(11):1059-1067.

15. Gordon PH, Moore DH, Miller RG, et al. Efficacy of minocyline in patients with amyotrophic lateral sclerosis: a phase III randomized trial. Lancet Neurol 2007;6(12):1045-1053.

16. Mitsumoto H, Brooks BR, Silani V. Clinical trials in amyotrophic lateral sclerosis: why so many negative trials and how can trials be improved? Lancet Neurol 2014;13(11):1127-1138.

17. Nicholson KA, Cudkowicz ME, Berry JD. Clinical trial designs in amyotrophic lateral sclerosis: does one design fit all? Neurotherapeutics 2015;12(2):376-383.

18. Mendez EF, Sattler R. Biomarker development for C9orf72 repeat expansion in ALS. Brain Res 2015;1607:26-35.

19. Turner MR, Kiernan MC, Leigh NP, Talbot K. Biomarkers in amyotrophic lateral sclerosis. Lancet Neurol 2009;8:94-109.

20. Su XW, Simmons Z, Mitchell RM, Kong L, Stephens HE, Connor JR. Biomarker-based predictive models for prognosis in amyotrophic lateral sclerosis. JAMA Neurol 2013;70(12):1505-1511.

21. Biomarkers Definitions Working Group. Biomarkers and surrogate endpoints: preferred definitions and conceptual framework. Clin Pharmacol Ther 2001;69(3):89-95.

22. Kruger T, Lautenschlager J, Grosskreutz J, Rhode H. Proteome analysis of body fluids for amyotrophic lateral sclerosis biomarker discovery. Proteomics Clin Appl 2013;7:123-135.

23. $\mathrm{Hu} \mathrm{S}$, Loo JA, Wong DT. Human body fluid proteome analysis. Proteomics 2006;6(23):6326-6353.

24. Rohlff C. Proteomics in molecular medicine: applications in central nervous systems disorders. Electrophoresis 2000;21:12271234.

25. Rothstein JD. Current hypotheses for the underlying biology of amyotrophic lateral sclerosis. Ann Neurol 2009;65(Suppl. 1):S3S9.

26. Xu Z, Cork LC, Griffin JW, Cleveland DW. Increased expression of neurofilament subunit NF-L produces morphological alterations that resemble the pathology of human motor neuron disease. Cell 1993;73(1):23-33.

27. Lee MK, Marszalek JR, Cleveland DW. A mutant neurofilament subunit causes massive, selective motor neuron death: implications for the pathogenesis of human motor neuron disease. Neuron 1994;13(4):975-988.

28. Brettschneider J, Petzold A, Sussmuth SD, Ludolph AC, Tumani $\mathrm{H}$. Axonal damage markers in cerebrospinal fluid are increased in ALS. Neurology 2006;66(6):852-856. 
29. Reijn TS, Abdo WF, Schelhaas HJ, Verbeek MM. CSF neurofilament protein analysis in the differential diagnosis of ALS. J Neurol 2009;256:615-619.

30. Ganesalingam J, An J, Bowser R, Andersen PM, Shaw CE. pNfH is a promising biomarker for ALS. Amyotroph Lateral Scler Frontotemporal Degener 2013;14(2):146-149.

31. Ganesalingam J, An J, Shaw CE, Shaw G, Lacomis D, Bowser R. Combination of neurofilament heavy chain and complement $\mathrm{C} 3$ as CSF biomarkers for ALS. J Neurochem 2011;117:528-537.

32. Lehnert S, Costa J, de Carvalho M, et al. Multicentre quality control evaluation of different biomarker candidates for amyotrophic lateral sclerosis. Amyotroph Lateral Scler Frontotemporal Degener 2014;15:344-350.

33. Oeckl P, Jardel C, Salachas F, et al. Multicenter validation of CSF neurofilaments as diagnostic biomarkers for ALS. Amyotroph Lateral Scler Frontotemporal Degener 2016;17(5-6):1-10.

34. Boylan KB, Glass JD, Crook JE, et al. Phosphorylated neurofilament heavy subunit (pNF-H) in peripheral blood and CSF as a potential prognostic biomarker in amyotrophic lateral sclerosis. J Neurol Neurosurg Psychiatry 2013;84(4):467-472.

35. Rosengren LE, Karlsson J-E, Karlsson J-O, Persson LI, Wikkelsø C. Patients with amyotrophic lateral sclerosis and other neurodegenerative diseases have increased levels of neurofilament protein in CSF. J Neurochem 1996;67(5):2013-2018.

36. Tortelli R, Ruggieri M, Cortese R, et al. Elevated cerebrospinal fluid neurofilament light levels in patients with amyotrophic lateral sclerosis: a possible marker of disease severity and progression. Eur J Neurol 2012;19(12):1561-1567.

37. Tortelli R, Copetti M, Ruggieri M, et al. Cerebrospinal fluid neurofilament light chain levels: marker of progression to generalized amyotrophic lateral sclerosis. Eur J Neurol 2015;22(1):215-218.

38. Lu CH, Macdonald-Wallis C, Gray E, et al. Neurofilament light chain: a prognostic biomarker in amyotrophic lateral sclerosis. Neurology 2015;84(22):2247-2257.

39. Menke RA, Gray E, Lu CH, et al. CSF neurofilament light chain reflects corticospinal tract degeneration in ALS. Ann Clin Transl Neurol 2015;2(7):748-755.

40. Steinacker P, Feneberg E, Weishaupt J, et al. Neurofilaments in the diagnosis of motoneuron diseases: a prospective study on 455 patients. J Neurol Neurosurg Psychiatry 2016;87:12-20.

41. Van Geel WJ, Rosengren LE, Verbeek MM. An enzyme immunoassay to quantify neurofilament light chain in cerebrospinal fluid. $\mathrm{J}$ Immunol Methods 2005;296:179-185.

42. Goldstein ME, Sternberger NH, Sternberger LA. Phosphorylation protects neurofilaments against proteolysis. J Neuroimmunol 1987;14(2):149-160.

43. McCombe PA, Pfluger C, Singh P, Lim CY, Airey C, Hernderson $\mathrm{RD}$. Serial measurements of phosphorylated neurofilament-heavy in the serum of subjects with amyotrophic lateral sclerosis. J Neurol Sci 2015;353:122-129.

44. Weydt P, Oeckl P, Huss A, et al. Neurofilament levels as biomarkers in asymptomatic and symptomatic familial amyotrophic lateral sclerosis. Ann Neurol 2016;79(1):152-158.

45. Ransohoff RM. How neuroinflammation contributes to neurodegeneration. Science 2016;353(6301):777-783.

46. McGeer PL, McGeer EG. Inflammatory processes in amyotrophic lateral sclerosis. Muscle Nerve 2002;26(4):459-470.

47. Chen Y, Liu XH, Wu JJ, et al. Proteomic analysis of cerebrospinal fluid in amyotrophic lateral sclerosis. Exp Ther Med 2016;11(6): 2095-2106

48. Varghese AM, Sharma A, Mishra P, et al. Chitotriosidase - a putative biomarker for sporadic amyotrophic lateral sclerosis. Clin Proteomics 2013;10(1):19.

49. Chen X, Chen Y, Wei Q, et al. Assessment of a multiple biomarker panel for diagnosis of amyotrophic lateral sclerosis. BMC Neurol 2016;16:173.
50. Moreau C, Gosset P, Brunaud-Danel V, et al. CSF profiles of angiogenic and inflammatory factors depend on the respiratory status of ALS patients. Amyotroph Lateral Scler 2009;10(3): 175-181.

51. Almer G, Teismann P, Stevic Z, et al. Increased levels of the proinflammatory prostaglandin PGE2 in CSF from ALS patients. Neurology 2002;58:1277-1279.

52. Ilzecka J. Prostaglandin E2 is increased in amyotrophic lateral sclerosis patients. Acta Neurol Scand 2003;108(2):125-129.

53. Mitchell RM, Freeman WM, Randazzo WT, et al. A CSF biomarker panel for identification of patients with amyotrophic lateral sclerosis. Neurology 2009;72:14-19.

54. Moreau C, Devos D, Brunaud-Danel V, et al. Elevated IL-6 and TNF-alpha levels in patients with ALS: inflammation or hypoxia? Neurology 2005;65(12):1958-1960.

55. Kuhle J, Lindberg RL, Regeniter A, et al. Increased levels of inflammatory chemokines in amyotrophic lateral sclerosis. Eur J Neurol 2009;16:771-774.

56. Lind AL, Wu D, Freyhult E, et al. A multiplex protein panel applied to cerebrospinal fluid reveals three new biomarker candidates in ALS but none in neuropathic pain patients. PLOS ONE 2016;11(2):e0149821.

57. Liu J, Gao L, Zang D. Elevated levels of IFN-gamma in CSF and serum of patients with amyotrophic lateral sclerosis. PLOS ONE 2015;10(9):e0136937.

58. Beers DR, Henkel JS, Zhao W, et al. Endogenous regulatory T lymphocytes ameliorate amyotrophic lateral sclerosis in mice and correlate with disease progression in patients with amyotrophic lateral sclerosis. Brain 2011;134(5):1293-1314.

59. Butovsky O, Siddiqui S, Gabriely G, et al. Modulating inflammatory monocytes with a unique microRNA gene signature ameliorates murine ALS. J Clin Invest 2012;122:3063-3087.

60. Schwartz M, Baruch K. The resolution of neuroinflammation in neurodegeneration: leukocyte recruitment via the choroid plexus. EMBO J 2014;33(1):7-22.

61. Smith R, Myers K, Ravits J, Bowser R. Amyotrophic lateral sclerosis: Is the spinal fluid pathway involved in seeding and spread? Med Hypotheses 2015;85(5):576-583.

62. DeJesus-Hernandez M, Mackenzie IR, Boeve BF, et al. Expanded GGGGCC hexanucleotide repeat in noncoding region of C9ORF72 causes chromosome 9p-linked FTD and ALS. Neuron 2011;72(2):245-256.

63. Renton AE, Majounie E, Waite A, et al. A hexanucleotide repeat expansion in C9ORF72 is the cause of chromosome 9p21-linked ALS-FTD. Neuron 2011;72(2):257-268.

64. Cleary JD, Ranum LPW. Repeat-associated non-ATG (RAN) translation in neurological disease. Hum Mol Genet 2013;22: R45-R51.

65. Ash PE, Bieniek KF, Gendron TF, et al. Unconventional translation of C9ORF72 GGGGCC expansion generates insoluble polypeptides specific to c9FTD/ALS. Neuron 2013;77(4):639-646.

66. Mori K, Weng S-M, Arzberger T, et al. The C9orf72 GGGGCC repeat is translated into aggregating dipeptide-repeat proteins in FTLD/ALS. Science 2013;339:1335-1338.

67. Wen X, Tan W, Westergard T, et al. Antisense proline-arginine RAN dipeptides linked to C9ORF72-ALS/FTD form toxic nuclear aggregates that initiate in vitro and in vivo neuronal death. Neuron 2014;84:1213-1225.

68. Westergard T, Jensen BK, Wen X, et al. Cell-to-cell transmission of dipeptide repeat proteins linked to C9orf72-ALS/FTD. Cell Rep 2016;17:645-652.

69. Mackenzie I, Arzberger T, Kremmer E, et al. Dipeptide repeat protein pathology in C9ORF72 mutation cases: clinico-pathological correlations. Acta Neuropathol 2013;126(6):859-879. 
70. Lee KH, Zhang P, Kim HJ, et al. C9orf72 dipeptide repeats impair the assembly, dynamics, and function of membrane-less organelles. Cell 2016;167(3):774-788.

71. Anderson P, Kedersha N. RNA granules: post-transcriptional and epigenetic modulators of gene expression. Nat Rev Mol Cell Biol 2009;10(6):430-436.

72. Gendron Tania F, Van Blitterswijk M, Bieniek KF, et al. Cerebellar c9RAN proteins associate with clinical and neuropathological characteristics of C9ORF72 repeat expansion carriers. Acta Neuropathol 2015;130:559-573.

73. Su Z, Zhang Y, Gendron TF, et al. Discovery of a biomarker and lead small molecules to target $\mathrm{r}(\mathrm{GGGGCC})$-associated defects in c9FTD/ALS. Neuron 2014;83(5):1043-1050.

74. King AE, Woodhouse A, Kirkcaldie MTK, Vickers JC. Excitotoxicity in ALS: overstimulation, or overreaction? Exp Neurol 2016;275:162-171.

75. Blasco H, Mavel S, Corcia P, Gordon PH. The glutamate hypothesis in ALS: pathophysiology and drug development. Curr Med Chem 2014;21(31):3551-3575.

76. Turner MR, Bowser R, Bruijn L, et al. Mechanisms, models and biomarkers in amyotrophic lateral sclerosis. Amyotroph Lateral Scler Frontotemporal Degener 2013;14(Suppl. 1):19-32.

77. Cid C, Alvarez-Cermeno JC, Regidor I, Salinas M, Alcazar A. Low concentrations of glutamate induce apoptosis in cultured neurons: implications for amyotrophic lateral sclerosis. J Neurol Sci 2003;206(1):91-95.

78. Spreux-Varoquaux O, Bensimon G, Lacomblez L, et al. Glutamate levels in cerebrospinal fluid in amyotrophic lateral sclerosis: a reappraisal using a new HPLC method with coulometric detection in a large cohort of patients. J Neurol Sci 2002;193(2):73-78.

79. Yanez M, Galan L, Matias-Guiu J, Vela A, Guerrero A, Garcia AG. CSF from amyotrophic lateral sclerosis patients produces glutamate independent death of rat motor brain cortical neurons: protection by resveratrol but not riluzole. Brain Res 2011;1423: 77-86.

80. Shaw PJ, Forrest V, Ince PG, Richardson JP, Wastell HJ. CSF and plasma amino acid levels in motor neuron disease: elevation of CSF glutamate in a subset of patients. Neurodegeneration 1995:4(2):209-216

81. Rothstein JD, Tsai G, Kuncl RW, et al. Abnormal excitatory amino acid metabolism in amyotrophic lateral sclerosis. Ann Neurol 1990;28(1):18-25.

82. Rothstein JD, Kuncl R, Chaudhry V, et al. Excitatory amino acids in amyotrophic lateral sclerosis: an update. Ann Neurol 1991;30(2):224-225.

83. Perry TL, Krieger C, Hansen S, Eisen A. Amyotrophic lateral sclerosis: amino acid levels in plasma and cerebrospinal fluid. Ann Neurol 1990;28(1):12-17.

84. Blin O, Samuel D, Nieoullon A, Serratice G. Changes in CSF amino acid concentrations during the evolution of amyotrophic lateral sclerosis. J Neurol Neurosurg Psychiatry 1994;57(1):119120.

85. Tikka TM, Vartiainen NE, Goldsteins G, et al. Minocycline prevents neurotoxicity induced by cerebrospinal fluid from patients with motor neurone disease. Brain 2002;125(Pt 4):722-731.

86. Wuolikainen A, Moritz T, Marklund SL, Antti H, Andersen PM. Disease-related changes in the cerebrospinal fluid metabolome in amyotrophic lateral sclerosis detected by GC/TOFMS. PLOS ONE 2011;6(4):e17947.

87. Wuolikainen A, Jonsson P, Ahnlund M, et al. Multi-platform mass spectrometry analysis of the CSF and plasma metabolomes of rigorously matched amyotrophic lateral sclerosis, Parkinson's disease and control subjects. Mol Biosyst 2016;12:1287-1298.

88. Bozik ME, Mitsumoto H, Brooks BR, et al. A post hoc analysis of subgroup outcomes and creatinine in the phase III clinical trial
(EMPOWER) of dexpramipexole in ALS. Amyotroph Lateral Scler Frontotemporal Degener 2014;15(5-6):406-413.

89. Neumann M, Sampathu DM, Kwong LK, et al. Ubiquitinated TDP-43 in frontotemporal lobar degeneration and amyotrophic lateral sclerosis. Science 2006;314(5796):130-133.

90. Arai T, Hasegawa M, Akiyama H, et al. TDP-43 is a component of ubiquitin-positive tau-negative inclusions in frontotemporal lobar degeneration and amyotrophic lateral sclerosis. Biochem Biophys Res Commun 2006;351:602-611.

91. Lagier-Tourenne C, Cleveland DW. Rethinking ALS: The FUS about TDP-43. Cell 2009;136:1001-1004.

92. Noto Y, Shibuya K, Sato Y, et al. Elevated CSF TDP-43 levels in amyotrophic lateral sclerosis: Specificity, sensitivity, and a possible prognostic value. Amyotroph Lateral Scler 2011;12(2):140143.

93. Kasai $\mathrm{T}$, Tokuda $\mathrm{T}$, Ishigami N, et al. Increased TDP-43 protein in cerebrospinal fluid of patients with amyotrophic lateral sclerosis. Acta Neuropathol 2009;117(1):55-62.

94. Junttila A, Kuvaja M, Hartikainen P, et al. Cerebrospinal fluid TDP-43 in frontotemporal lobar degeneration and amyotrophic lateral sclerosis patients with and without the C9ORF72 hexanucleotide expansion. Dement Geriatr Cogn Disord Extra 2016;6:142-149.

95. Steinacker P, Hendrich C, Sperfeld AD, et al. TDP-43 in cerebrospinal fluid of patients with frontotemporal lobar degeneration and amyotrophic lateral sclerosis. Arch Neurol 2008;65(11):14811487.

96. Xiao S, Sanelli T, Chiang H, et al. Low molecular weight species of TDP-43 generated by abnormal splicing form inclusions in amyotrophic lateral sclerosis and result in motor neuron death. Acta Neuropathol 2015;130(1):49-61.

97. Ranganathan S, Williams E, Ganchev P, et al. Proteomic profiling of cerebrospinal fluid identifies biomarkers for amyotrophic lateral sclerosis. J Neurochem 2005;95:1461-1471.

98. Ryberg H, An J, Darko S, et al. Discovery and verification of amyotrophic lateral sclerosis biomarkers by mass spectrometry based proteomics. Muscle Nerve 2010;42:104-111.

99. Pasinetti GM, Ungar LH, Lange DJ, et al. Identification of potential CSF biomarkers in ALS. Neurology 2006;66:1218-1222.

100. Tsuji-Akimoto S, Yabe I, Niino M, Kikuchi S, Sasaki H. Cystatin $\mathrm{C}$ in cerebrospinal fluid as a biomarker of ALS. Neurosci Lett 2009;452(1):52-55.

101. Paraoan L, Grierson I. Focus on molecules: cystatin C. Exp Eye Res 2007;84:1019-1020.

102. Nagai A, Terashima M, Sheikh AM, et al. Involvement of cystatin C in pathophysiology of CNS diseases. Front Biosci 2008;13: 3470-3479.

103. Okamoto K, Hirai S, Amari M, Watanabe M, Sakurai A. Bunina bodies in amyotrophic lateral sclerosis immunostained with rabbit anti-cystatin C serum. Neurosci Lett 1993;162(1-2):125-128.

104. Mori F, Tanji K, Miki Y, Wakabayashi K. Decreased cystatin C immunoreactivity in spinal motor neurons and astrocytes in amyotrophic lateral sclerosis. J Neuropathol Exp Neurol 2009;68(11): 1200-1206.

105. Wilson ME, Boumaza I, Bowser R. Cystatin C: A candidate biomarker for amyotrophic lateral sclerosis. PLOS ONE 2010;5(12): e15133.

106. Wilson ME, Boumaza I, Bowser R. Measurement of cystatin C functional activity in the cerebrospinal fluid of amyotrophic lateral sclerosis and control subjects. Fluids Barriers CNS 2013;10:15.

107. Bartel DP. MicroRNAs: genomics, biogenesis, mechanism, and function. Cell 2004;116:281-297.

108. Bartel DP. MicroRNAs: target recognition and regulatory functions. Cell 2009;136:215-233.

109. Eitan C, Hornstein E. Vulnerability of microRNA biogenesis in FTD-ALS. Brain Res 2016;1647:105-111. 
110. Kocerha J, Kauppinen S, Wahlestedt C. microRNAs in CNS disorders. Neuromol Med 2009;11(3):162-172.

111. Weinberg MS, Wood MJ. Short non-coding RNA biology and neurodegenerative disorders: novel disease targets and therapeutics. Hum Mol Genet 2009;18(R1):R27-R39.

112. Goodall EF, Heath PR, Bandmann O, Kirby J, Shaw PJ. Neuronal dark matter: the emerging role of microRNAs in neurodegeneration. Front Cell Neurosci 2013;7:178.

113. Haramati S, Chapnik E, Sztainberg Y, et al. miRNA malfunction causes spinal motor neuron disease. Proc Natl Acad Sci U S A 2010;107(29):13111-13116.

114. Campos-Melo D, Droppelmann CA, He Z, Volkening K, Strong MJ. Altered microRNA expression profile in amyotrophic lateral sclerosis: a role in the regulation of NFL mRNA levels. Mol Brain 2013;6:26.

115. Emde A, Eitan C, Liou LL, et al. Dysregulated miRNA biogenesis downstream of cellular stress and ALS-causing mutations: a new mechanism for ALS. EMBOJ 2015;34:2633-2651.

116. Benigni M, Ricci C, Jones AR, Giannini F, Al-Chalabi A, Battistini S. Identification of miRNAs as potential biomarkers in cerebrospinal fluid from amyotrophic lateral sclerosis patients. Neuromolecular Med 2016;18:551-560.

117. Freischmidt A, Muller K, Ludolph AC, Weishaupt JH. Systemic dysregulation of TDP-43 binding microRNAs in amyotrophic lateral sclerosis. Acta Neuropathol Commun 2013;1:42.

118. De Felice B, Annunziata A, Fiorentino G, et al. miR-338-3p is over-expressed in blood, CFS, serum and spinal cord from sporadic amyotrophic lateral sclerosis patients. Neurogenetics 2014;15(4):243-253.

119. Freischmidt A, Muller K, Zondler L, et al. Serum microRNAs in sporadic amyotrophic lateral sclerosis. Neurobiol Aging 2015;36(9):2660.

120. Takahashi I, Hama Y, Matsushima M, et al. Identification of plasma microRNAs as a biomarker of sporadic amyotrophic lateral sclerosis. Mol Brain 2015;8(1):67.

121. Bunton-Stasyshyn RK, Saccon RA, Fratta P, Fisher EM. SOD1 function and its implications for amyotrophic lateral sclerosis pathology: new and renascent themes. Neuroscientist 2015;21(5): 519-529.

122. Andersen PM, Sims KB, Xin WW, et al. Sixteen novel mutations in the $\mathrm{Cu} / \mathrm{Zn}$ superoxide dismutase gene in amyotrophic lateral sclerosis: a decade of discoveries, defects and disputes. Amyotroph Lateral Scler Other Motor Neuron Disord 2003;4(2): 62-73.

123. Kaur SJ, McKeown SR, Rashid S. Mutant SOD1 mediated pathogenesis of amyotrophic lateral sclerosis. Gene 2016;577(2):109118.

124. Frutiger K, Lukas TJ, Gorrie G, Ajroud-Driss S, Siddique T. Gender difference in levels of $\mathrm{Cu} / \mathrm{Zn}$ superoxide dismutase (SOD1) in cerebrospinal fluid of patients with amyotrophic lateral sclerosis. Amyotroph Lateral Scler 2008;9(3):184-187.

125. Miller TM, Kaspar BK, Kops GJ, et al. Virus-delivered small RNA silencing sustains strength in amyotrophic lateral sclerosis. Ann Neurol 2005;57(5):773-776.

126. Ralph GS, Radcliffe PA, Day DM, et al. Silencing mutant SOD1 using RNAi protects against neurodegeneration and extends survival in an ALS model. Nat Med 2005;11(4):429-433.

127. Lange DJ, Andersen PM, Remanan R, Marklund S, Benjamin D. Pyrimethamine decreases levels of SOD1 in leukocytes and cerebrospinal fluid of ALS patients: a phase I pilot study. Amyotroph Lateral Scler Frontotemporal Degener 2013;14:199-204.

128. Winer L, Srinivasan D, Chun S, et al. SOD1 in cerebral spinal fluid as a pharmacodynamic marker for antisense oligonucleotide therapy. JAMA Neurol 2013;70:201-207.

129. Miller TM, Pestronk A, David W, et al. An antisense oligonucleotide against SOD1 delivered intrathecally for patients with SOD1 familial amyotrophic lateral sclerosis: a phase 1 , randomised, firstin-man study. Lancet Neurol 2013;12(5):435-442.

130. Johanson CE, Stopa EG, McMillan PN. The blood-cerebrospinal fluid barrier: structure and functional significance. Methods Mol Biol 2011;686:101-131.

131. Spector R, Keep RF, Robert Snodgrass S, Smith QR, Johanson CE. A balanced view of choroid plexus structure and function: focus on adult humans. Exp Neurol 2015;267:78-86.

132. Boylan K, Yang C, Crook J, et al. Immunoreactivity of the phosphorylated axonal neurofilament $\mathrm{H}$ subunit ( $\mathrm{pNF}-\mathrm{H})$ in blood of ALS model rodents and ALS patients: evaluation of blood pNF-H as a potential ALS biomarker. J Neurochem 2009;111(5):1182-1191.

133. de Andrade HM, de Albuquerque M, Avansini SH, et al. MicroRNAs-424 and 206 are potential prognostic markers in spinal onset amyotrophic lateral sclerosis. J Neurol Sci 2016;368:19-24.

134. Ehrhart J, Smith AJ, Kuzmin-Nichols N, et al. Humoral factors in ALS patients during disease progression. J Neuroinflammation 2015;12:127.

135. Houi K, Kobayashi T, Kato S, Mochio S, Inoue K. Increased plasma TGF-beta1 in patients with amyotrophic lateral sclerosis. Acta Neurol Scand 2002;106(5):299-301.

136. Ilzecka J, Stelmasiak Z, Dobosz B. Transforming growth factorBeta 1 (tgf-Beta 1) in patients with amyotrophic lateral sclerosis. Cytokine 2002;20(5):239-243.

137. Lu CH, Allen K, Oei F, et al. Systemic inflammatory response and neuromuscular involvement in amyotrophic lateral sclerosis. Neurol Neuroimmunol Neuroinflammation 2016;3(4):e244.

138. Cereda C, Baiocchi C, Bongioanni P, et al. TNF and sTNFR1/2 plasma levels in ALS patients. J Neuroimmunol 2008;194(1-2): 123-131.

139. Zonder L, Muller K, Khalaji S, et al. Peripheral monocytes are functionally altered and invade the CNS in ALS patients. Acta Neuropathol 2016;132(3):391-411.

140. Henkel JS, Beers DR, Wen S, et al. Regulatory T-lymphocytes mediate amyotrophic lateral sclerosis progression and survival. EMBO Mol Med 2013;5:64-79.

141. Verstraete E, Kuiperij HB, van Blitterswijk MM, et al. TDP-43 plasma levels are higher in amyotrophic lateral sclerosis. Amyotroph Lateral Scler 2012;13(5):446-451.

142. Suarez-Calvet M, Dols-Icardo O, Llado A, et al. Plasma phosphorylated TDP-43 levels are elevated in patients with frontotemporal dementia carrying a C9orf72 repeat expansion or a GRN mutation. J Neurol Neurosurg Psychiatry 2014;85(6):684-691.

143. De Marco G, Lupino E, Calvo A, et al. Cytoplasmic accumulation of TDP-43 in circulating lymphomonocytes of ALS patients with and without TARDBP mutations. Acta Neuropathol 2011;121(5): 611-622.

144. Cecchi M, Messina $\mathrm{P}$, Airoldi L, et al. Plasma amino acids patterns and age of onset of amyotrophic lateral sclerosis. Amyotroph Lateral Scler Frontotemporal Degener 2014;15(5-6):371-375.

145. Andreadou E, Kapaki E, Kokotis P, et al. Plasma glutamate and glycine levels in patients with amyotrophic lateral sclerosis. In Vivo 2008;22(1):137-141.

146. Andreadou E, Kapaki E, Kokotis P, et al. Plasma glutamate and glycine levels in patients with amyotrophic lateral sclerosis: the effect of riluzole treatment. Clin Neurol Neurosurg 2008;110(3): 222-226.

147. Niebroj-Dobosz I, Janik P, Kwiecinski H. Effect of Riluzole on serum amino acids in patients with amyotrophic lateral sclerosis. Acta Neurol Scand 2002;106(1):39-43.

148. Paganoni S, Zhang M, Quiroz Zarate A, et al. Uric acid levels predict survival in men with amyotrophic lateral sclerosis. J Neurol 2012;259(9):1923-1928. 
149. Lawton KA, Brown MV, Alexander D, et al. Plasma metabolomic biomarker panel to distinguish patients with amyotrophic lateral sclerosis from disease mimics. Amyotroph Lateral Scler Frontotemporal Degener 2014;15(5-6):362-370.

150. Schutzer SE, Liu T, Natelson BH, et al. Establishing the proteome of normal human cerebrospinal fluid. PLOS ONE 2010;5(6): e10980.

151. Zhang J, Goodlett DR, Peskind ER, et al. Quantitative proteomic analysis of age-related changes in human cerebrospinal fluid. Neurobiol Aging 2005;26(2):207-227.

152. Shepheard SR, Chataway T, Schultz DW, Rush RA, Rogers ML. The extracellular domain of neurotrophin receptor $\mathrm{p} 75$ as a candidate biomarker for amyotrophic lateral sclerosis. PLOS ONE 2014;9(1):9.

153. Ono S, Shimizu N, Imai T, Rodriguez GP. Urinary collagen metabolite excretion in amyotrophic lateral sclerosis. Muscle Nerve 2001;24:821-825.

154. Ono S, Imai T, Matsubara S, et al. Decreased urinary concentrations of type IV collagen in amyotrophic lateral sclerosis. Acta Neurol Scand 1999;100(2):111-116.

155. Bogdanov M, Brown RH, Matson WR, et al. Increased oxidative damage to DNA in ALS patients. Free Radical Biol Med 2000;29(7):652-658

156. Mitsumoto H, Santella RM, Liu X, et al. Oxidative stress biomarkers in sporadic ALS. Amyotroph Lateral Scler 2008;9(3): 177-183.

157. Ono S, Imai T, Munakata S, et al. Collagen abnormalities in the spinal cord from patients with amyotrophic lateral sclerosis. J Neurol Sci 1998;160(2):140-147.

158. Obayashi K, Sato K, Shimazaki R, et al. Salivary chromogranin A: useful and quantitative biochemical marker of affective state in patients with amyotrophic lateral sclerosis. Intern Med 2008;47(21):1875-1879.

159. Roozendaal B, Kim S, Wolf OT, Kim MS, Sung KK, Lee S. The cortisol awakening response in amyotrophic lateral sclerosis is blunted and correlates with clinical status and depressive mood. Psychoneuroendocrinology 2012;37(1):20-26.

160. Maier A, Deigendesch N, Muller K, et al. Interleukin-1 antagonist anakinra in amyotrophic lateral sclerosis - a pilot study. PLOS ONE 2015;10(10):e0139684.

161. Fiala M, Mizwicki MT, Weitzman R, Magpantay L, Nishimoto N. Tocilizumab infusion therapy normalizes inflammation in sporadic ALS patients. Am J Neurodegener Dis 2013;2(2):129-139.

162. Mizwicki MT, Fiala M, Magpantay L, et al. Tocilizumab attenuates inflammation in ALS patients through inhibition of IL6 receptor signaling. Am J Neurodegener Dis 2012;1(3):305-315.
163. Lu CH, Petzold A, Kalmar B, Dick J, Malaspina A, Greensmith L. Plasma neurofilament heavy chain levels correlate to markers of late stage disease progression and treatment response in SOD1(G93A) mice that model ALS. PLOS ONE 2012;7(7): e40998.

164. Stommel EW, Cohen JA, Fadul CE, et al. Efficacy of thalidomide for the treatment of amyotrophic lateral sclerosis: a phase II open label clinical trial. Amyotroph Lateral Scler 2009;10(5-6):393404.

165. Levine TD, Bowser R, Hank N, Saperstein D. A pilot trial of memantine and riluzole in ALS: correlation to CSF biomarkers. Amyotroph Lateral Scler 2010;11(6):514-519.

166. Cudkowicz ME, Shefner JM, Schoenfeld DA, et al. Trial of celecoxib in amyotrophic lateral sclerosis. Ann Neurol 2006;60(1):22-31.

167. Bakkar N, Boehringer A, Bowser R. Use of biomarkers in ALS drug development and clinical trials. Brain Res 2015;1607:94107.

168. Sussmuth SD, Sperfeld AD, Hinz A, et al. CSF glial markers correlate with survival in amyotrophic lateral sclerosis. Neurology 2010;74:982-987.

169. Lu CH, Petzold A, Topping J, et al. Plasma neurofilament heavy chain levels and disease progression in amyotrophic lateral sclerosis: insights from a longitudinal study. J Neurol Neurosurg Psychiatry 2015;86(5):565-573.

170. Blasco H, Patin F, Madji Hounoum B, et al. Metabolomics in amyotrophic lateral sclerosis: how far can it take us? Eur J Neurol 2016;23(3):447-454

171. Gray E, Larkin JR, Claridge TD, Talbot K, Sibson NR, Turner MR. The longitudinal cerebrospinal fluid metabolomic profile of amyotrophic lateral sclerosis. Amyotroph Lateral Scler Frontotemporal Degener 2015;16(7-8):456-463.

172. Blasco H, Corcia P, Pradat PF, et al. Metabolomics in cerebrospinal fluid of patients with amyotrophic lateral sclerosis: an untargeted approach via high-resolution mass spectrometry. J Proteome Res 2013;12(8):3746-3754.

173. Lawton KA, Cudkowicz ME, Brown MV, et al. Biochemical alterations associated with ALS. Amyotroph Lateral Scler 2012;13(1):110-118.

174. Kumar A, Bala L, Kalita J, et al. Metabolomic analysis of serum by (1) H NMR spectroscopy in amyotrophic lateral sclerosis. Clin Chim Acta 2010;411(7-8):563-567.

175. Dettmer K, Aronov PA, Hammock BD. Mass spectrometry-based metabolomics. Mass Spectrom Rev 2007;26(1):51-78.

176. Wishart DS, Jewison T, Guo AC, et al. HMDB 3.0-The Human Metabolome Database in 2013. Nucl Acids Res 2013;41:D801D807. 